\author{
Accelerator Division \\ Alternating Gradient Synchrotron Department \\ BROOKHAVEN NATIONAL LABORATORY \\ Associated Universities, Inc. \\ Upton, New York 11973 \\ Accelerator Division \\ Technical Note \\ AGS/AD/Tech. Note No. 285
}

\title{
A COMPUTER PROGRAM TO CALCULATE THE IMPEDANCE FUNCTION \\ OF THE VHF CAVITY AND DE-QING SWITCH
}

J.M. Brennan and D. Kasha

August 20, 1987

\section{Introduction}

A high frequency cavity will be installed in the AGS ring and used to make a controlled blow-up of the longitudinal phase space area. The lower density thus obtained will allow a beam of higher intensity to be accelerated through the gamma-transition with negligible particle loss. This cavity must satisfy two conflicting requirements of the gap impedance: a high impedance when it is operating and low impedance when it is not. When the cavity is in operation, during the blow-up period of $50 \mathrm{~ms}$, it must provide a large voltage (a significant fraction of the main rf voltage per turn). The large voltage requires a high impedance. But when the cavity is not in operation, it should offer as low an impedance as possible to the beam in order to not stimulate longitudinal instabilities.

These conflicting requirements will be satisfied by a switched de-Qing of an otherwise high Q cavity. The switching will be accomplished by an array of PIN diodes which are tightly coupled to the cavity. The diodes terminate a resonant line and switch between a short circuit and a matched load. The short circuit transforms to an open at the cavity and the matched load loads the cavity heavily.

Since these two resonance structures interact strongly, it is necessary to study the coupled circuit in detail in order to predict the behavior of the cavity. The program VHFPIN was written to facilitate the study. This paper describes the theoretical principles used in the calculation and the procedure for using the program. In addition, a series of laboratory measurements were made on a test structure, analogous to the cavity-switch circuit, to check the validity of 
the program's results. The results of the laboratory measurements and a typical calculation for the real cavity are reported.

\section{Theory}

The system model consists of two coaxial lines as shown schematically in Figure 1. Line 1 is shorted at one end, open at the other end, and at a distance $l_{1}$ from the open end, a second line is tapped into the line in a " $\mathrm{T}$ " configuration. The particle beam travels through the center conductor of line 1 , so that it crosses the open end and is accelerated by the voltage that may be present. The impedance at the open end of line 1 is proportional to the impedance seen by the beam. The proportionality constant will depend on how the cavity is coupled to the power amplifier. A line stretcher between the power amplifier and the cavity has been incorporated into the design of the VHF cavity system. It will be used to adjust the proportionality constant by transforming the output impedance of the power amplifier to the lowest possible value as seen from the cavity gap.

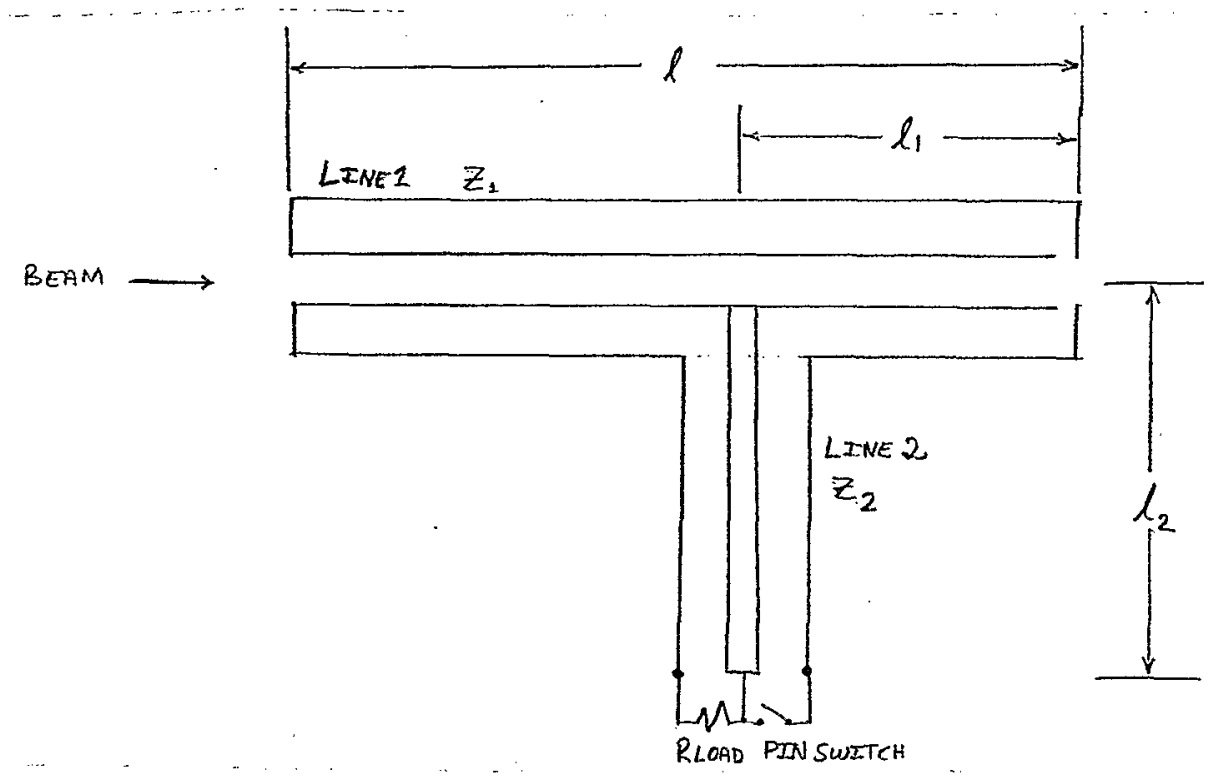

Figure 1 -- VHF Cavity Model.

The second line is termianted by a resistive load (rload) and a PIN diode switch in parallel with the load, allowing the total termination of line 2 to be a near short or the load resistance, depending on the bias of the PIN diode switch. The PIN diode switch consists of $N$ diodes in parallel and a bypass capacitor, Cbp (to isolate the d.c. bias current needed to close the switch), in series with the diodes. 
The PIN diode switch models for the "on" (forward bias) and "off" (reverse bias) states are shown in Figure 2.

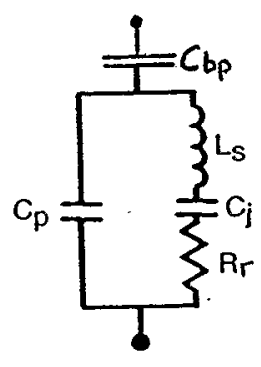

a) REVERSE BIAS

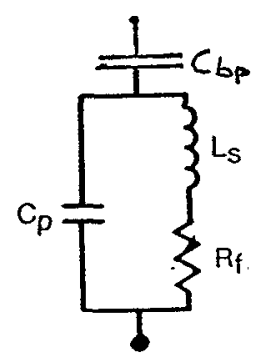

b) FORWARD BIAS

$$
\begin{aligned}
& \mathrm{Cp}=\text { Package Capacitance } \\
& \mathrm{Ls}=\text { Lead Inductance } \\
& \mathrm{Cj}=\text { Junction Capacitance } \\
& \mathrm{Rr}=\text { Diode Reistance in Reverse Bias } \\
& \mathrm{Rf}=\text { Diode Resistance in Forward Mode } \\
& \mathrm{Cbp}=\text { Bypass Capacitance }
\end{aligned}
$$

\section{Figure 2 -- PIN Switch Model (1).}

The cavity impedance is calculated by transforming the termination of the lines through their respective lengths to the tap point. The transformed impedances are combined (in parallel) and the resultant impedance is transformed to the open end of line 1 to find the cavity impedance. The line's characteristic impedance $\left(z_{0}\right)$ and the propagation constant $(\gamma)$ must be determined. A transmission line with inner conductor of radius, a (meters); outer conductor of radius, b (meters); with no dielectric; and surface resistance $\mathrm{rs}\left(=2.6^{-7}\right.$ (f) $1 / 2 \mathrm{ohm}$ for copper) has a characteristic impedance, $\mathrm{z}_{0}$ :

$$
\mathrm{Z}_{\mathrm{o}}=60 \ln (\mathrm{b} / \mathrm{a}) \mathrm{ohm}
$$

and propagation constant

$$
\begin{gathered}
\gamma=\alpha+i k \text {, where } \\
\alpha=(r s /(2 n))(l / b+\ell / a) / \ln (b / a)
\end{gathered}
$$


$\eta$ is the impedance of free space $(=377 \mathrm{ohm})$

$$
k=2 \pi / \lambda
$$

$\lambda$ is the free space wavelength $(=c / f)$

The general transformation equation looking into a transmission line of characteristic impedance $Z_{o}$, with termination $Z_{r}$ and length 1 (in meters) is:(2)

$$
z_{\text {in }}=z_{o}\left(z_{r}+z_{o} \tanh (\gamma \ell)\right) /\left(z_{o}+z_{r} \tanh (\gamma \ell)\right) .
$$

For this model, the termination on line 2 is transformed over the length of line 2 to find its impedance at the tap point. The short $\left(z_{r}=0\right)$ at the end of line 1 is transformed over length $l-l_{1}$ of Line 1 to find its impedance at the tap point. These two impedances are combined in parallel and transformed over the length $\ell_{l}$ to find the final cavity impedance.

The cavity $Q$ is calcualted from the derivative of the cavity reactance $(\mathrm{x})$.

$$
Q=\frac{f_{o}}{2 R}\left(\frac{d x}{d f}\right)
$$

where $\mathrm{dx} / \mathrm{df}$ is the derivative of the cavity reactance an $R$ is the cavity resistance, at the resonance frequency $f_{o}$.

\section{Laboratory Measurements}

The program was tested against a model VHF cavity and de-Qing line constructed from 3-1/8" coaxial line components. The $Q$ of a high-Q cavity is more amenable to direct measurement than the impedance, whereas, for a low $Q$ cavity the impedance is more easily measured. In these measurements, the Q's were measured in the high $Q$ cases and the impedance in the low $Q$ cases. The $Q^{\prime}$ 's were measured by the transmission method, using the HP-8505A network analyzer, and the impedances were measured by direct connection of the HP-4815A vector impedance meter to the open end of the center conductor. 
For the $Q$ measurements, the cavity was driven with an inductive loop near the shorted end. A dual directional coupler in the drive line was used to measure the forward and reflected voltage waves in order to determine the reflection coefficient and VSWR. The cavity voltage was sampled with a weakly coupled capacitive probe at the open end. The loaded $Q\left(Q_{\ell}\right)$ was deduced from the bandwidth at the halfpower frequencies $(-3 \mathrm{db}$ points). Two tap points at $1 / 2$ and $1 / 4$ of the length of line 1 (from the shorted end) were measured.

The unloaded cavity $Q\left(Q_{O}\right)$ is determined from $Q_{\ell}$ using the coupling coefficient, $\beta$,

$$
Q_{0}=(1+\beta) Q_{\ell}
$$

$\beta$ can be found from the VSWR,

$$
\begin{gathered}
\beta=1 / \text { VSWR if the cavity is undercoupled } \\
\beta=\text { VSWR if the cavity is overcoupled }
\end{gathered}
$$

The degree of coupling was determined from the polar display on the HP-8505A of the input reflection coefficient. Figure 3 shows typical plots for the overcoupled, undercoupled, and critically coupled cases.

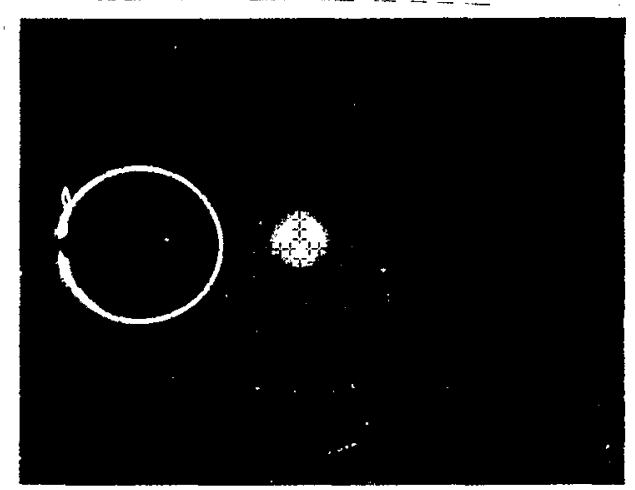

a) Under coupled

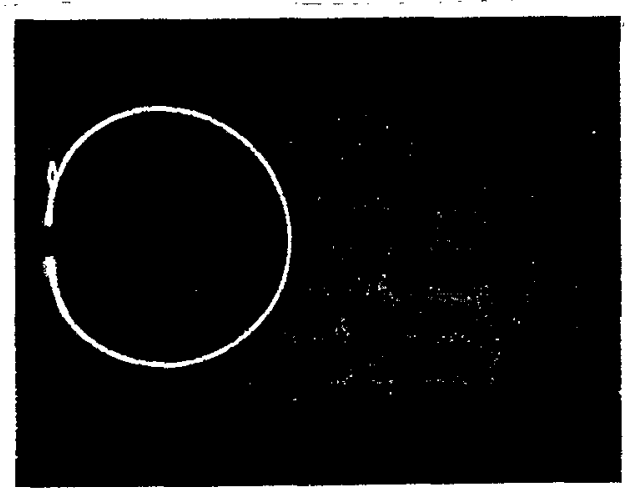

b) Critical coupling

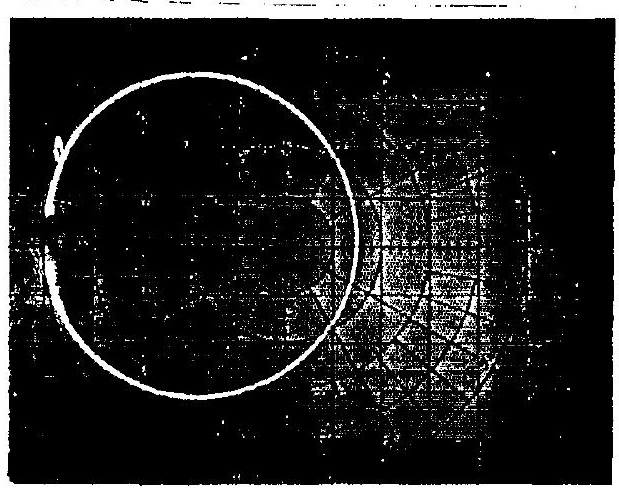

c) Over coupled

Figure 3 -- Polar display showing cavity coupling.

In the overcoupled case, the angle of the reflection coefficient is zero because the input impedance of the cavity is higher than the source impedance (i.e., the cavity resembles an open). In the undercoupled case, the angle is $180^{\circ}$. The VSWR is determined from the reflection coefficient by

$$
\operatorname{VSWR}=(1+|\rho|) /(1-|\rho|) \text {. }
$$


In the cavity's de-Qed state, $\beta$ is essentially zero since the cavity impedance seen at the drive loop is very low compared to the source impedance.

The resonance of line 2 (a $3 / 4$ wavelength section) was found to be 95.7 MHz. Line 1 was tuned as close as possible to this frequency (system stability was $0.5 \mathrm{MHz}$ ) with a sliding short. The model's line characteristics are calculated using inner (a) and outer (b) radii:

$$
\begin{array}{lll}
a_{1}=0.0167 \mathrm{~m} & b_{1}=0.0381 \mathrm{~m} & 1=0.78 \mathrm{~m} \\
a_{2}=0.0111 \mathrm{~m} & b_{2}=0.0381 \mathrm{~m} & 1_{2}=2.35 \mathrm{~m}
\end{array}
$$

Line 2 was terminated either in a short, 50 ohms or 75 ohms, the latter being the characteristic impedance of line 2 .

The measured and calculated $Q^{\prime} s$ and impedances are:

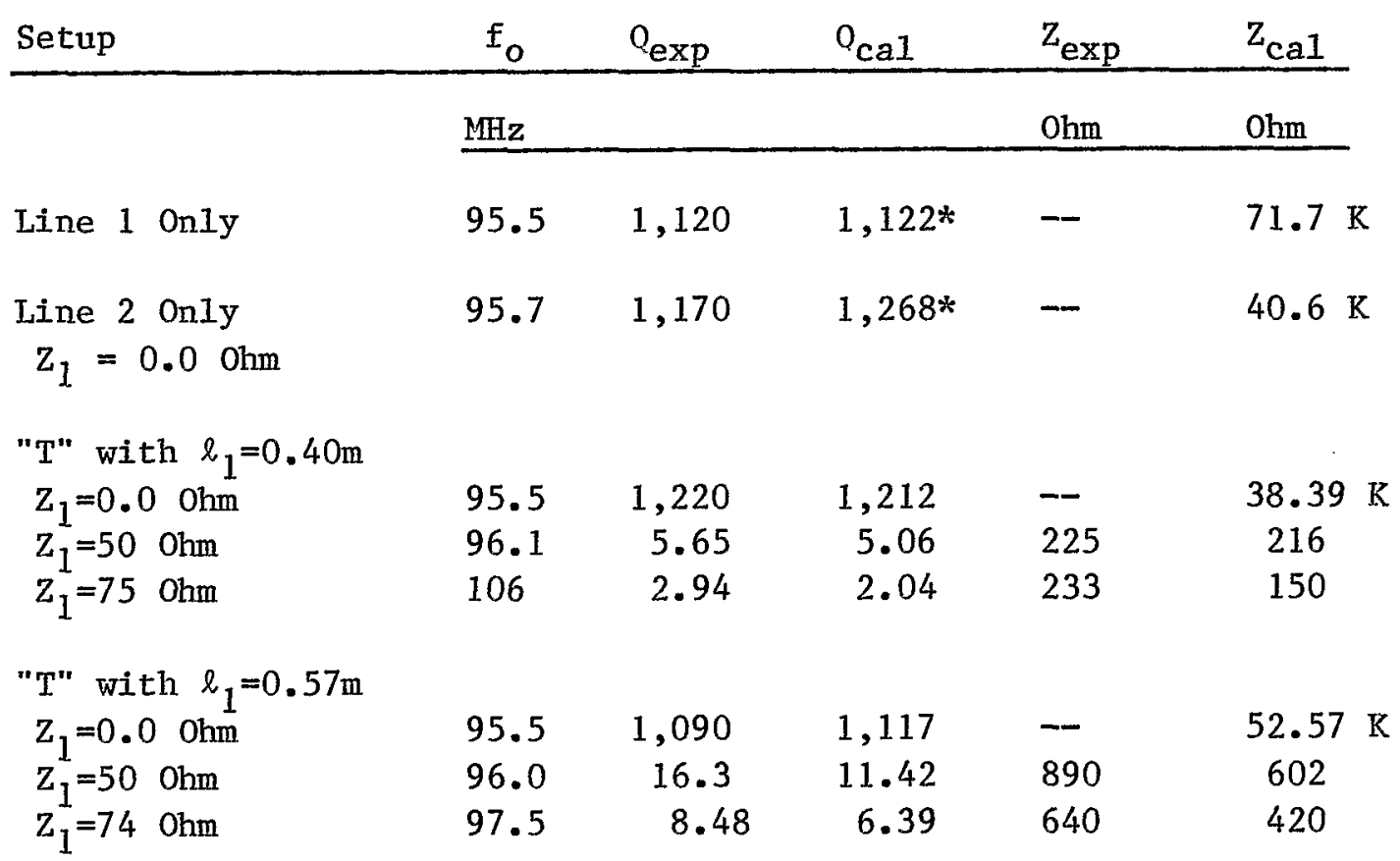

* Fit to measured Q by adjusting loss factors. 
Two factors were introduced in the program to compensate for losses in the lines, loss_factorl and loss_factor2. These were adjusted to match the measured and calculated Q's of the individual lines. The program was run with the loss factors shown below and with the PIN diode switch parameters chosen to approximate a perfect switch.

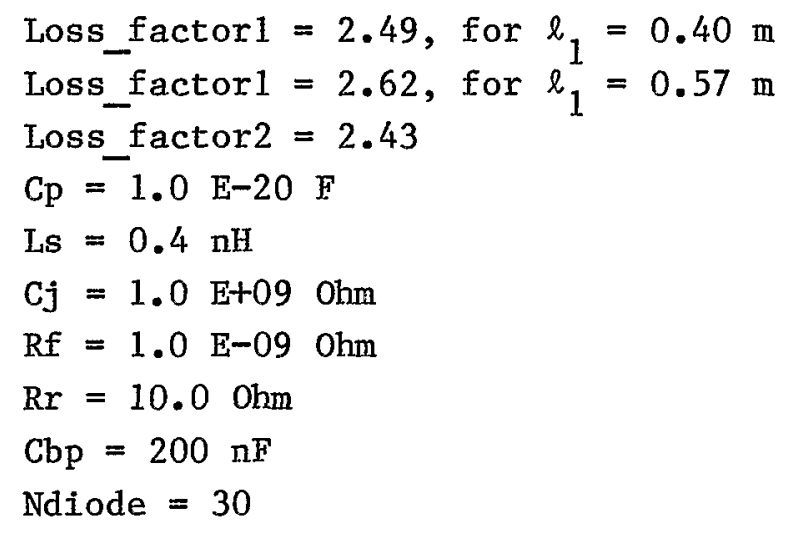

\section{Design Problem}

The program was used to help choose the proper distance $l_{1}$ from the tap point to the accelerating gap. The location of the tap point determines the gap impedance in both the high-Q and low-Q state. This is true in the high $Q$ state because the switch assembly is a resonant structure with a $Q$ considerably lower than the cavity intrinsic $Q$. The $Q$ of the combined structure, and therefore the gap impedance, is given by the coupling strength (tap point) between the two structures. In the low-Q state, the non-resonant low impedance of the load is coupled more strongly to the gap by reducing $\ell_{1}$.

It is useful to have a means of optimizing the $Q$ in the high-Q state in order to adjust th bandwidth of the cavity to accommodate the phase modulation program which will be used in bunch dilution.

Another consideration in the choice of the value for $l_{1}$ is the current carrying capability of PIN diodes. The value of the current is given by

$$
I=\left(\nabla_{\text {gap }} / Z_{0}\right) \sin \left((\pi / 2)\left(\ell-\ell_{1}\right) / \ell\right)
$$

Where:

$\mathrm{Z}_{\mathrm{o}}$ is the characteristic impedance of Line 2 .

$\ell$ is the cavity length.

$\mathrm{V}_{\mathrm{g}}$ is the gap voltage.

The number of diodes must be chosen to safely handle this current. 
The cavity and PIN switch parameters are shown below. The switch parameters are best estimates of values for real switch.

$$
\begin{aligned}
& f_{0}=91.5 \mathrm{MHz} \\
& a_{1}=0.0841 \mathrm{~m} \\
& b_{1}=0.152 \mathrm{~m} \\
& a_{2}=0.0167 \mathrm{~m} \\
& b_{2}=0.0381 \mathrm{~m} \\
& \mathrm{rs}=2.6 \times 10^{-7} \mathrm{Ohm} \\
& \text { loss_factorl }=1 \\
& \text { loss_factor2 }=1 \\
& \text { rload }=50 \mathrm{Ohm} \\
& \text { ndiode }=20 \\
& \mathrm{Cbp}=200 \mathrm{nF} \\
& \mathrm{Rf}=0.3 \mathrm{Ohm} \\
& \mathrm{Rr}=10 \mathrm{Ohm} \\
& \mathrm{Ls}=0.4 \mathrm{nH} \\
& \mathrm{Cp}-0.18 \mathrm{pF} \\
& \mathrm{Cj}=0.7 \mathrm{pF}
\end{aligned}
$$

\begin{tabular}{|c|c|c|c|c|c|c|}
\hline${ }^{{ }}{ }_{1}$ & $\mathrm{Zf}$ & $\mathrm{Zr}$ & $\mathrm{Zf} / \mathrm{Zr}$ & Qf & Qr & $\mathrm{Qf} / \mathrm{Qr}$ \\
\hline$\underline{\mathrm{m}}$ & Ohm & $\mathrm{Ohm}$ & & & & \\
\hline 0.1 & $40.4 \mathrm{~K}$ & 85.2 & 474 & 2,730 & 4.71 & 580 \\
\hline 0.2 & $44.7 \mathrm{~K}$ & 96.8 & 461 & 2,810 & 5.05 & 556 \\
\hline 0.3 & $53.4 \mathrm{~K}$ & 120 & 445 & 2,950 & 5.45 & 541 \\
\hline 0.4 & $69.3 \mathrm{~K}$ & 166 & 417 & 3,210 & 6.30 & 510 \\
\hline 0.5 & $99.3 \mathrm{~K}$ & 258 & 384 & 3,700 & 8.09 & 457 \\
\hline 0.6 & $160.0 \mathrm{~K}$ & 482 & 332 & 4,620 & 12.6 & 367 \\
\hline 0.7 & $280.0 \mathrm{~K}$ & $1.38 \mathrm{~K}$ & 203 & 6,190 & 30.6 & 202 \\
\hline 0.8 & $408.0 \mathrm{~K}$ & $39.9 \mathrm{~K}$ & 10.2 & 7,480 & 798 & 9.4 \\
\hline
\end{tabular}

The $91.5 \mathrm{MHz}$ nominal cavity frequency corresponds to a $1 / 4$ wavelength of $0.820 \mathrm{~m}$. The cavity impedance and $Q$ in the two states were calculated with $\ell_{1}$ ranging from 0.1 to $0.8 \mathrm{~m}$. The results are shown below ( $f$ for the forward bias state, high-Q, and $r$ for the reverse bias state, $10 w-Q)$.

The proper loss factors for this cavity cannot be determined before laboratory tests of the cavity, so the impedance and $Q$ values should 
not be used as absolute values. The ratios $\mathrm{Zf} / \mathrm{Zr}$ and $\mathrm{Qf} / \mathrm{Qr}$ show the relative difference between states, and are to a high degree independent of the loss factors. A tap point of $\ell_{1}=0.7 \mathrm{~m}$ shows the de-Qing desired, and corresponds to a voltage at the tap point of 0.23

$\mathrm{V}_{\mathrm{g}}$. The impedance in the forward state changes with the tap point because the PIN diode switch is not a perfect short, nor is the line lossless, and therefore does not transform to an infinite impedance at the tap point. Increasing Ndiode and/or Cbp would make a more perfect switch.

\section{Program Procedure}

The program runs on the VAX Fortran system and requires the main file, VHFPIN.EXE, and a data file in namelist format which the user specifies during the run. Appendix A shows the format of the datafile. To run the program enter:

\$run vhfpin

The program then asks:

type input file name

Enter datafile name, for example: VHFPIN.DAT.

From this point, the program offers the option to change any of the parameters, first the frequency range, then the cavity parameters and finally the switch parameters. The changes made are retained until the user exits the program. After the changes are made, the program displays the frequency, cavity impedance and impedance phase angle over the range selected. Also, to help in the PIN switch design, the switch impedance and total termination of line 2 are displayed at the resonance frequency. After the frequency sweep, if a resonance was found (determined from a zero crossing of the phase angle) the cavity $Q$ is calculated and displayed. After the run, the program offers to create a datafile of the run. If a file output is desired, the program then prompts the user for a filename and a comment to head the file. It then asks if another run is desired. To load a different datafile, the program must be exited and restarted. 


\section{Conclusion}

The measured Q's and impedances of the model VHF cavity agree well with the computed values. The one fitted parameter of the calculation, the loss factor, will normally be of much less significance for the design of a real cavity, such as the VHF cavity. For a real cavity, as opposed to the model measured here, the more rigorous construction techniques would imply that the measured $Q$ would be closer to the calculated $Q$. The real usefulness of the program comes from its ability to compute the relative change in gap impedance as a function of the location of the tap point and the consequences of an imperfect PIN diode switch.

\section{References}

1. M/A-COM Silicon Products, "PIN Diode Designers' Guide", 1 South Avenue, Burlington, MA 01803, p. 7.

2. Marshall, S., Electromagnetic Concepts and Applications, PrenticeHa11, Inc., Englewood Cliffs, NJ, 1987, p. 372.

3. Ginzton, E., Microwave Measurements, McGraw-Hill Book Company, Inc., NY, 1957, p. 397, 407. 
Appendix A

Sample data file for VHFPIN.FOR

\$indat1

fzero $=91.5 \mathrm{e} 6$,

fs tart $=91.3 \mathrm{e} 6$,

fs top $=91.7 \mathrm{e}$,

nstep=100, $\$$

Sindat2

$\mathrm{n} 1=1$.,

$\mathrm{n} 2=3$.,

$r I 1=.40$,

a1 $=8.41 e-2$,

b1 $=.152$,

a2 $=1.67 \mathrm{e}-2$,

b2 $=3.81 e-2$,

rho $=2.6 e-7$,

loss factor $1=1$.,

loss_factor2 $=1 ., \$$

Sindat3

rload $=50$, ,

ndi ode $=20$,

$\mathrm{cbp}=200 \cdot \mathrm{e}-9$,

mode $=$ ' forward',

$\mathrm{rf}=.3$,

$\mathrm{rs}=10$.

ls $=.4 \mathrm{e}-9$,

$\mathrm{cp}=1.8 \mathrm{e}-13$,

$c j=7 . e-13, \quad s$ 